\title{
Bayes' rule for clinicians: an introduction
}

\section{Chris F. Westbury*}

Department of Psychology, University of Alberta, Edmonton, AB, Canada

Edited by:

Edward Callus, IRCCS Policlinico San

Donato, Italy

Reviewed by:

Gianluca Castelnuovo, Università

Cattolica del Sacro Cuore, Italy

Gian Mauro Manzoni, Istituto

Auxologico Italiano IRCCS, Italy

Francesco Pagnini, University of

Bergamo, Italy

*Correspondence:

Chris F. Westbury, Department of

Psychology, University of Alberta, P220

Biological Sciences Building,

Edmonton, AB, Canada, T6G 2E9.

e-mail: chrisw@ualberta.ca
Bayes' Rule is a way of calculating conditional probabilities. It is difficult to find an explanation of its relevance that is both mathematically comprehensive and easily accessible to all readers. This article tries to fill that void, by laying out the nature of Bayes' Rule and its implications for clinicians in a way that assumes little or no background in probability theory. It builds on Meehl and Rosen's (1955) classic paper, by laying out algebraic proofs that they simply allude to, and by providing extremely simple and intuitively accessible examples of the concepts that they assumed their reader understood.

Keywords: probability, diagnosis, Bayes theory, base rates
Bayes' Rule is a way of calculating conditional probabilities. Although it is simple in its conception, Baye's Rule can be fiendishly difficult for beginners to understand and apply. In part this is because it forces us to confront and overcome strong biases in our natural way of thinking and in part it is because it is not easy to be specific about exactly where Bayes' Rule will apply, or how it may apply in any particular case. The purpose of this paper is to present and explore the simplest forms of Bayes' Rule, and to explain how it may be used in practical reasoning, especially in clinical settings.

A great deal has been written about the importance of conditional probability in diagnostic situations. However, there are, so far as I know, no papers that are both comprehensive and simple. Most writing on the topic, particularly in probability textbooks, assumes too much knowledge of probability for diagnosticians, losing the clinical reader by alluding to simple proofs without giving them. Many introductory psychometrics textbooks err on the other side, either ignoring conditional probability altogether, or by considering it in such a cursory manner that the reader has little chance to understand what it is and why it matters. This paper is intended to fill the void between simplicity and thoroughness. The exposition provided here assumes only the most basic understanding of non-conditional probability, and provides both concrete examples and simple algebraic proofs of some implications of Bayes' Rule that are clinically relevant. It may be reasonably considered an interpretive guide to perhaps the best introduction to Bayes' Rule for the clinician, Meehl and Rosen's classic (1955) paper, Antecedent probability and the efficiency of psychometric signs, patterns, or cutting scores. Many readers find this paper very difficult to understand, in part because the authors do make mathematical claims without providing any detailed explanation of where they came from. The present paper frames Meehl and Rosen's claims with a much more basic introduction than they give, and fills in some simple proofs to which they only allude.
The first section consists of a general introduction to understanding conditional probabilities. The second section introduces Bayes' Rule itself, in an historical and mathematical setting. The third section lays out some implications of Bayes' Rule that follow as a direct result of its definition.

\section{CONDITIONAL PROBABILITIES}

Conditional probabilities are those probabilities whose value depends on the value of another probability. Such probabilities are ubiquitous. For example, we may wish to calculate the probability that a particular patient has a disease, given the presence of a particular set of symptoms. The probability of disease may be more or less close to certain, depending on the nature and number of symptoms. We will certainly wish to take into account a patient's relevant prior history with medication (e.g., the known probability of responding) before prescribing medication (Belmaker et al., 2010). Or we may wish to take into account factors (such as defensiveness) that might impact on a success in psychotherapy before we begin that therapy (Zanarini et al., 2009). More generally, restating all these specific cases in a more abstract way, we may wish to calculate the probability that a given hypothesis is true, given a diverse set of evidence (say, results from several diagnostic instruments) for or against it. Hypothesis testing is just one way of assigning weight to belief. Conditional probabilities come into play when we wish to decide how much confidence we wish to assign to a given such beliefs as "this patient will respond to this intervention," or "this person should receive this specific diagnosis" or "it is worth incorporating this method into my clinical practice."

A very simple example of conditional probability will elucidate its nature. Consider the question: How likely is that you would win the jackpot in a lottery if you didn't have a lottery ticket? It should be obvious that the answer is zero - you certainly could not win if you didn't even have a ticket. It may be equally obvious that you are more likely to win the lottery the more tickets you buy. So the probability of winning a lottery is really a conditional probability, 
where your odds of winning are conditional on the number of tickets you have purchased. If you have zero tickets, then you have no chance of winning. With one ticket, you have a small chance to win. With two tickets, your odds will be twice as good.

We symbolize conditionality by using a vertical slash "|", which can be read as "given." Then the odds of winning a lottery with one ticket could be expressed as $P$ (Winning | One ticket). There are many "keywords" in a problem's definition that may (but need not necessarily) suggest that you are dealing with a problem of conditional probability. Phrases like "given," "if," "with the constraint that," "assuming that," "under the assumption that" and so on all suggest that there may be a conditional clause in the problem.

One thing that sometimes confuses students of probability is the fact that all probability problems are really conditional. Consider the simple probability question: "What is the probability of getting a head with a coin toss?" The question implicitly assumes that the coin is fair (that is, that heads and tails are equally probable), and should really be phrased "What is the probability of getting a head with a coin toss, given that the coin is fair?" Non-conditional probability problems conceal their conditional clause in the background assumptions that either explicitly or implicitly limit the domain in which the probability calculation is supposed to apply.

This observation sheds light on what conditionality actually does. A condition always serves exactly this role: to limit the domain in which the "non-conditional" portion of the question is supposed to apply. When you are asked "What is the probability of getting a head with a coin toss?" you are supposed to understand that we are limiting the domain to which the question applies by considering only fair coins. When you are asked "What is the probability that you have disease $\mathrm{X}$, given that you have symptom Y?, you are supposed to understand that the probability calculation only applies to those people who do have symptom Y. An appropriate way of thinking about conditional probability is to understand that a conditional limits the number and kind of cases you are supposed to consider. You can think of the vertical slash as meaning something like "ignoring everything to which the following constraint does not apply." So "What is the probability of getting a head with a coin toss, given that the coin is fair?" means "What is the probability of getting a head with a coin toss, ignoring every coin to which the following statement does not apply: The coin is fair."

Bayes' Rule and other methods of solving conditional probability questions are simply mathematical means of limiting the domain across which a calculation is being computed. To see that this is so, consider the following simple question:

Three tall and two short men went on a picnic with four tall and four short woman. What is $P$ (Tall | Female), the probability that a person is tall, given that the person is female?

The solution to this problem may be immediately obvious, but it is worth working through a few ways of solving it. These are all formally the same, though they may appear to be different.

The first way is just to turn the question into a very simple non-conditional question that we know how to solve. Following the discussion above, the question can be re-phrased to say "What is the probability that a person is tall, ignoring everyone who is not a woman?" If we ignore the men, we have a really simple question, viz. "Four tall and four short woman went on a picnic? What is the probability that a woman who went on the picnic was tall?" This is simple (that is, non-conditional) probability. Like any simple probability question, it can be solved by dividing the number of ways the outcome of interest ("being tall") can happen by the number of ways any outcome in the domain ("being a woman") can happen. So: 4 tall women $/(4$ tall woman +4 short woman $)=0.5$ probability that a person on the picnic was tall, given that she was a woman.

A formally identical way of solving the same problem can be seen by drawing a $2 \times 2$ table such as the following:

\begin{tabular}{lll}
\hline Gender/Height & Female & Male \\
\hline Tall & 4 & 3 \\
Short & 4 & 2 \\
\hline
\end{tabular}

The condition "Given that she was a female" means that we can simply ignore the rightmost column of this box, the males, and act as if the question about the probability of being tall only applied to the leftmost column, the woman.

Here comes the tricky part. This diagram makes clear what the question is asking: What is the ratio of people who are both tall and female (top left cell) to people who are female (sum of left column)? We can re-state this and solve the problem in a third way by asking: What is the ratio of the probability that a person is both female and tall to the probability that a person is female? To see why, consider the concrete example again. There were 13 people on the picnic. Since 4 were tall females, the probability of being a tall female is $4 / 13$. Since 8 were females, the probability of being female was $8 / 13$. The ratio of people who were both tall and female to people who were female is therefore $4 / 13$ / $8 / 13$, or $4 / 8$, or $50 \%$. The reason this may seem "tricky" is that here we consider the domain as a whole - all people who went on the picnic- and then take the ratio of two subsets within that domain.

If you understand this third method of calculating the conditional probability, then you will understand Bayes' Rule. Bayes Rule is a way to "automatically" pick out this very same ratio: the ratio of the probability of being in the cell of interest (in this case, the cell consisting of tall and female picnickers) to the probability of being in the sub-domain of interest that is specified by the conditional clause (in this case, woman, a subset of all the people who went on the picnic).

Before we look at how the math works, let's introduce the rule itself.

\section{BAYES' RULE}

Bayes' Rule is very often referred to Bayes' Theorem, but it is not really a theorem, and should more properly be referred to as Bayes' Rule (Hacking, 2001). In either case, it is so-called because it was first stated (in a different form than we consider here) by Reverend Thomas Bayes in his "Essay toward solving a problem in the doctrine of chances," which was published in the Philosophical Transactions of the Royal Society of London in 1764. Bayes was a minister interested in probability and stated a form of his famous rule in the context of solving a somewhat complex problem involving billiard balls that need not concern us here.

Bayes' Rule has many analogous forms of varying degrees of apparent complexity. This paper concerns itself almost entirely with the simplest form, which covers the cases in which two sets of 
mutually exclusive possibilities A and B are considered, and where the total probability in each set is 1 . At the end of the paper we will briefly examine how this most simple case is just a specific case of a more general form of Bayes' Rule. The simplest case covers many diagnostic situations, in which the patient either has or does not have a diagnosable condition (possibility set $\mathrm{A}$ ) and either has or does not have a set of symptoms (possibility set B). For such cases, Bayes' Rule can be used to calculate $P(\mathrm{~A} \mid \mathrm{B})$, the probability that the patient has the condition given the symptom set. Bayes' Rule says that:

$P(\mathrm{~A} \mid \mathrm{B})=P(\mathrm{~B} \mid \mathrm{A}) P(\mathrm{~A}) / P(\mathrm{~B})$

$P(\mathrm{~A})$ is called the marginal or prior probability of $A$, since it is the probability of A prior to having any information about B. Similarly, the term $P(B)$ is the marginal or prior probability of $B$. Because it does depend on having information about $\mathrm{B}$, the term $P(\mathrm{~A} \mid \mathrm{B})$ is called the posterior probability of $A$ given $B$. The term $P(B \mid A)$ is called the likelihood function for $B$ given $A$.

In the third solution to the example above, we solve for the probability of being female, given that you are tall, by considered the ratio of those who were tall and female to those who were female:

\section{$P($ Tall $\mid$ Female $)=P($ Tall \& Female $) / P($ Female $)$}

This suggests that Bayes' Rule can also be stated in the following form:

$P(\mathrm{~A} \mid \mathrm{B})=P(\mathrm{~A} \& \mathrm{~B}) / P(\mathrm{~B})$

From this it should be evident, by equating the numerators of the two equations above, that:

$P(\mathrm{~A} \& \mathrm{~B})=P(\mathrm{~B} \mid \mathrm{A}) P(\mathrm{~A})$

This is true by the definition of " $\&$." Let us try to understand why this is so, by again considering the three tall and two short men went on a picnic with four tall and four short woman. We have already convinced ourselves that $P$ (Female \& tall) is $4 / 13$, because there are 4 people in the cell of interest and thirteen people in the problem's domain. Let's see how the definition agrees with this answer. The definition above says that $P($ Female $\&$ Tall $)=P($ Tall $\mid$ Female $) \mathrm{P}($ Female $) . P($ Tall $\mid$ Female $)$, the probability of a picnicker being tall given that she is female, is $4 / 8$. $P$ (Female) is $8 / 13$, because eight of the thirteen people on the picnic are females. $4 / 8$ multiplied by $8 / 13$ is $4 / 13$.

Note that it is equally correct to write that:

$P(\mathrm{~A} \& \mathrm{~B})=P(\mathrm{~A} \mid \mathrm{B}) P(\mathrm{~B})$

In other words:

$P(\mathrm{~B} \mid \mathrm{A}) P(\mathrm{~A})=P(\mathrm{~A} \mid \mathrm{B}) P(\mathrm{~B})$

Let's see why using the same example. Now we will see that $P($ Female $\&$ Tall $)=P($ Female $\mid$ Tall $) P($ Tall $) . P($ Female $\mid$ Tall $)$, the probability of a picnicker being female given that he or she is tall, is $4 / 7$, because there are four tall females and seven tall people altogether. $P$ (Tall) is $7 / 13$, because seven of the 13 people on the picnic are tall. $4 / 7$ multiplied by $7 / 13$ is $4 / 13$.

If you go back and look at the $2 \times 2$ table above, you should be able to understand why these two calculations of $P(\mathrm{~A} \& \mathrm{~B})$ must be the same. The first calculation picks out the cell of tall females by column. The second picks it out by row. It doesn't matter if you concern yourself with females who are tall or tall people who are females - in the end you must get to the same answer if you want to know about people who are both tall and female. A tall female person is also a female tall person.

So now we have

$P(\mathrm{~A} \mid \mathrm{B})=P(\mathrm{~B} \mid \mathrm{A}) P(\mathrm{~A}) / P(\mathrm{~B})=P(\mathrm{~A} \mid \mathrm{B}) P(\mathrm{~B}) / P(\mathrm{~B})$

Although either form will give the same answer, the first form is the "canonical" form of Bayes' Rule, for a reason that should be obvious: because the second form contains the same element on the right, $P(\mathrm{~A} \mid \mathrm{B})$, as the left element that we are trying to calculate. If we already know $P(\mathrm{~A} \mid \mathrm{B})$, then we don't need to compute it. If we don't know it, then it will not help us to include it in the equation we will use to calculate it.

Bayes' Rule can be easily derived from the definition of $P(A \mid B)$, in the following manner:

1. $P(\mathrm{~A} \mid \mathrm{B})=P(\mathrm{~A} \& \mathrm{~B}) / P(\mathrm{~B})[$ By definition]

2. $P(\mathrm{~B} \mid \mathrm{A})=P(\mathrm{~A} \& \mathrm{~B}) / P(\mathrm{~A})[$ By definition]

3. $P(\mathrm{~B} \mid \mathrm{A}) P(\mathrm{~A})=P(\mathrm{~A} \& \mathrm{~B})[$ Multiply 2.) by $P(\mathrm{~A})]$

4. $P(\mathrm{~A} \mid \mathrm{B}) P(\mathrm{~B})=P(\mathrm{~B} \mid \mathrm{A}) P(\mathrm{~A})[$ Substitute 1.) in 3.) ]

5. $P(\mathrm{~A} \mid \mathrm{B})=P(\mathrm{~B} \mid \mathrm{A}) P(\mathrm{~A}) / P(\mathrm{~B})[$ Bayes' Rule]

It might seem at first glance that Bayes' Rule cannot be a very helpful rule, because it says that to solve a conditional probability $P(\mathrm{~A} \mid \mathrm{B})$ you have to know another conditional probability $P(\mathrm{~B} \mid \mathrm{A})$. However, Reverend Bayes' insight was that in many cases the second possibility is knowable when the first is not. In diagnostic cases where were are trying to calculate $P$ (Condition | Symptom) we often know $P$ (Symptom | Condition), the probability that you have the symptom given the condition, because this data has been collected from previous confirmed cases.

\section{IMPLICATIONS OF BAYES RULE}

Bayes' Rule is very simple. However, its implications are often unexpected. Many studies have shown that people of all kinds - even those who are trained in probability theory - tend to be very poor at estimating conditional probabilities. It seems to be kind of innate incompetence in our species. As a result, people are often surprised by what Bayes' Rule tells them.

Let us consider a concrete example given in Meehl and Rosen (1955), from which much of the discussion in this section is drawn. A particular disorder has a base rate occurrence of $1 / 1000$ people. A test to detect this disease has a false positive rate of 5\% - that is, $5 \%$ of the time that it says a person has the disease, it is mistaken. Assume that the false negative rate is $0 \%$ - the test correctly diagnoses every person who does have the disease. What is the chance that a randomly selected person with a positive result actually has the disease?

When this question was posed to Harvard University medical students, about half said that the answer was $95 \%$, presumably because the test has a $5 \%$ false positive rate. The average response was $56 \%$. Only $16 \%$ gave the correct answer, which can be computed with Bayes' Rule in the following manner:

Let: $P(\mathrm{~A})=$ Probability of having the disease $=0.001$

$P(\mathrm{~B})=$ Probability of positive test

$=$ Sum of probabilities of all independent ways to get a positive test 
$=$ Probability of true positive + probability of false positive

$=$ (True positive base rate $\times$ Percent correctly identi-

fied $)+($ Negative Base Rate $\times$ Percent incorrectly identified $)$

$=(0.001 \times 1)+(0.999 \times 0.05)$

$=0.051$

$P(\mathrm{~B} \mid \mathrm{A})=$ Probability of positive test given disease $=1$

Then: $P(\mathrm{~A} \mid \mathrm{B})=P(\mathrm{~B} \mid \mathrm{A}) P(\mathrm{~A}) / P(\mathrm{~B})$

$=(1 \times 0.001) /(0.051)$

$=0.02$, or $2 \%$

Although the test is highly accurate, it in fact gives a correct positive result just $2 \%$ of the time. How can this be? The answer (and the importance of Bayes' Rule in diagnostic situations) lies in the highly skewed base rates of the disease. Since so few people actually have the disease, the probability of a true positive test result is very small. It is swamped by the probability of a false positive result, which is fifty times larger than the probability of a true positive result.

You can concretely understand how the false positive rate swamps the true positive rate by considering a population of 10,000 people who are given the test. Just $1 / 1000$ th or 10 of those people will actually have the disease and therefore a true positive test result. However, 5\% of the remaining 9990 people, or 500 people, will have a false positive test result. So the probability that a person has the disease given that they have a positive test result is $10 / 510$, or $2 \%$.

Many cases are subtle. Consider another case cited by Meehl and Rosen (1955). This involved a test to detect psychological adjustment in soldiers. The authors of the instrument validated their test by giving it to 415 soldiers known to be well-adjusted, and 89 soldiers known to be mal-adjusted. The test correctly diagnosed $55 \%$ of the mal-adjusted soldiers as mal-adjusted, and incorrectly diagnosed only $19 \%$ of the adjusted soldiers. Since the true positive rate (55\%) is much higher than the false positive rate (19\%), the authors believed their test was good. However, they failed to take into account base rates. Meehl and Rosen did not know $P$ (Maladjusted), the probability that a randomly selected soldier was maladjusted, but they guessed that it might be as high as $5 \%$. With this estimate, we can use Bayes' Rule as follows:

Let $P(\mathrm{M})=$ Probability of being maladjusted $=0.05$, by assumption

Let $P(\mathrm{D})=$ Probability of being diagnosed as being maladjusted.

$=$ Probability of true positive + probability of false positive

$=($ True positive base rate $\times$ Percent correctly identified $)+($ Negative Base Rate $\times$ Percent incorrectly identified $)$

$=(0.55 \times 0.05)+(0.95 \times 0.19)$

$=0.208$

$P(\mathrm{D} \mid \mathrm{M})=$ Probability of being diagnosed, given maladjustment. $=0.55$, as found by the authors.

$P(\mathrm{M} \mid \mathrm{D})=$ Probability of maladjustment given diagnosis as maladjusted

$$
\begin{aligned}
& =P(\mathrm{D} \mid \mathrm{M}) P(\mathrm{M}) / P(\mathrm{D}) \text { [Bayes' Rule] } \\
& =(0.55)(0.05) / 0.208 \\
& =0.13 \text { or } 13 \%
\end{aligned}
$$

When base rates are taken into account, the test's true positive rate is just $13 \%$, not $55 \%$ as claimed. The test is still better than guessing that everyone is maladjusted. With that strategy $5 \%$ of positive diagnoses would be correct. However, note that the test's diagnosis of maladjustment is much more likely to be wrong $(87 \%$ probability) than right (13\% probability).

Of course clinicians prefer to make diagnoses that are more likely to be right than wrong. We can state this desire more formally by saying that we prefer the fraction of the population that is diagnosed correctly to be greater than the fraction of the population that is diagnosed incorrectly. Mathematically this leads to a useful conclusion in the following manner:

Fraction diagnosed correctly $>$ Fraction diagnosed incorrectly

Fraction diagnosed incorrectly / Fraction diagnosed correctly $<1$

Let $\mathrm{D}=$ Diseased and $\mathrm{S}=$ Selected (“ $\sim$ " means "not")

$P(\mathrm{D} \& \sim \mathrm{S}) / P(\mathrm{D} \& \mathrm{~S})<1$ [Substitute symbols]

$P(\mathrm{D} \mid \sim \mathrm{S}) P(\sim \mathrm{S}) / P(\mathrm{D} \mid \mathrm{S}) P(\mathrm{~S})<1$ [By definition of “\&”]

$P(\mathrm{D} \mid \sim \mathrm{S}) / P(\mathrm{D} \mid \mathrm{S}) P(\mathrm{~S})<1 / P(\sim \mathrm{S})[$ Divide by $P(\sim \mathrm{S})$ ]

$P(\mathrm{D} \mid \sim \mathrm{S}) / P(\mathrm{D} \mid \mathrm{S})<P(\mathrm{~S}) / P(\sim \mathrm{S})$ [Multiply by $P(\mathrm{~S})$ ]

In English this can be expressed as:

False positive rate/True positive rate $<$ Positive base rate/Negative base rate

We need the ratio of positive to negative base rates to be greater than the ratio of the false positive rate to the true positive rate, if we want to be more likely to be right than wrong.

This can be a handy heuristic because it allows us to calculate the minimum proportion of the population we are working with that needs to be diseased in order for our diagnostic methods to be useful. In the example above, the ratio of false positive to true positive rates is $0.19 / 0.55$ or 0.34 . This means that the test can only be useful - in the sense of having a positive diagnosis that is more likely to be true than false - when it is used in settings in which the ratio of the maladjusted people (positive base rate) to the number of people who are not maladjusted (negative base rate) is at least 0.34 .

Again we can consider one example from Meehl and Rosen (1955). Imagine that you have a test that correctly identifies $80 \%$ of brain-damaged patients, but also misidentifies $15 \%$ of non-braindamaged people. The calculation above says that this test will only be reliable if the ratio of brain-damaged to non-brain-damaged people is greater than $0.15 / 0.80$, or about 0.19 . If we are using the test in a setting which has a lower ratio of brain damaged people, we will run in to the problem described above, in which we find that the base rates have made it more likely that we are wrong than right when we make a diagnosis.

As another example, let us consider an analysis of the utility of the screening version of the Psychopathy Checklist (PCL:SV; Hart et al., 1995) in predicting violence within a year after discharge from a civil psychiatric institute. Skeem and Mulvey (2001) report that "a threshold of approximately 8 [much lower than the cut off of 17 for probable diagnoses of psychopathy] simultaneously maximizes the sensitivity and specificity of the PCL:SV in predicting violence in this sample" (p. 365). They therefore suggest 8 as the optimal cutoff. With that cut-off, the test has a true positive rate (sensitivity) of 0.72 . It has a true negative rate (specificity) of 0.65 , and therefore a false positive rate of 0.35 . The ratio of false to true positives is thus 
$0.35 / 0.72$, or 0.486 . With the prescribed cut-off point, the test will only predict violence correctly if at least $48.6 \%$ of people in the sample are violent. In the sample, $245 / 871$ or $28 \%$ were actually violent. A person would be more accurate than using the cut-off if she simply guessed that no one will be violent, since she would then correctly classify the $72 \%$ of the discharged who will not be. With a higher cut-off of 16 , the true positive rate is just 0.21 but the false positive rate plummets from 0.35 to 0.06 . This gives a ratio of false to true positives of $0.06 / 0.21$ or 0.286 , close to the actual ratio of violent individuals in the population, suggesting this (or a higher) cut-off point is better from the point of view of maximizing accuracy. In this case, the mathematical result is somewhat equivocal because of the unequal costs of making false positive and false negative identifications. The rate of identifying future violence is certainly very poor with the prescribed cut-off of 8 . The ratio of false to true positives shows that if a person uses this cut-off, he will do only a little better than he would if he predicted who will be violent by flipping a coin, since using the cut-off will make him wrong (48.6\% of time) almost as often as he is right ( $51.4 \%$ of the time). However, it may be more desirable to err on the side of conservatism by incorrectly treating $35 \%$ of people as likely to be violent than to lower the overall error rate (by raising the cutoff above 16) at the cost of missing $79 \%$ of the people who actually will be violent. Sometimes we have pragmatic reasons to prefer one kind of inaccuracy to another.

Note that Meehl's heuristic does not mean that the true population base rate must be as high as the calculation prescribes - it is sufficient for the base rate of the subpopulation to which the test is exposed to be high enough. If the test is used in settings (such a mental clinic to which front-line physicians refer) that have "higher concentration" of maladjusted subjects than the general population as a result on non-random sampling of that population, then the test may be useful in that setting, even though it may not be reliable if subjects were randomly selected from the population as a whole. For example, Fontaine et al. (2001) looked at how an elevated $t$-score on the Minnesota Multiphasic Personality Inventory-Adolescent (MMPI-A; Butcher et al., 1992) was able to classify subjects as "normal" or "clinical" in an inpatient sample with a base rate of $50 \%$ versus a normative sample with a base rate of $20 \%$. They found, as Bayes' Rule guarantees they must, that "the classification accuracy hit rates generally increased as the clinical base rate increased from 20 to $50 \%$ of the total sample" (p. 276).

This ability to skew true diagnosis rates in a favorable direction by pre-selecting subjects has important implications. In most of the examples we have considered so far, we have assumed low base rates. If the base rates are very high, an opposite issue arises: it becomes increasingly less worthwhile to give a diagnostic test if the base rate odds of the diagnosis are very high to begin with, because test results may add so little certainty to the base rate as to make it not worth the effort (or risk) of administering the tests. A recent practical example with a very strong result concerns the use of the Wada test, an invasive, potentially dangerous, and expensive test for determining language lateralization prior to surgery. The test involves injecting sodium amytal into each internal carotid artery to anesthetize each cerebral hemisphere independently. Kemp et al. (2008) looked at 141 consecutive administrations of the Wada test. One key finding was that no patient failed the test who had both a right temporal lesion and a stronger verbal than visual memory test result. The memory test result is also a key piece of lateralizing evidence (suggesting left lateralized language) that can be obtained relatively cheaply and safely. Based on the base rate information for this particular subset of patients with right temporal lesions and clear memory test results, Kemp et al. concluded that "that this group of patients is at negligible risk of failing the Wada test and the risks of the procedure probably outweigh the information obtained" (p. 632).

This is one "degenerate" case in which the base rate in one subsample of interest went $100 \%$ in one direction, eliminating the possibility that another test could add any further certainty to the diagnostic question of language lateralization. The degenerate case in the opposite direction - when base rates are $0 \%$ - has equally clear implications: except perhaps as a confirmation of the continuing absence of the disease in a population, it is a waste of resources to test for a condition that no one has. In between 0 and $100 \%$, the implications of a conditional clause, such as a the probability of that a person has a disease given a positive tests results, become more severe as the base rates moves away from 0.5 in either direction. The further the base rate is from 50/50, the further it takes the posterior probability $P(\mathrm{~A} \mid \mathrm{B})$ from the simple "hit rate," given by taking the ratio of the true positive rate to the positive diagnoses rate (the sum of the true and false positive rate).

Mathematically, we can see this by expanding the canonical form of Bayes' Rule given above, just as we did with the example of the maladjusted soldiers above:

Let $P(\mathrm{C})=$ Probability of belonging to the diagnostic category

Let $\mathrm{TP}=$ True positive rate $=P(\mathrm{C} \&$ Diagnosed $)$

Let $\mathrm{FP}=$ False positive rate $=P(\sim \mathrm{C} \&$ Diagnosed $)$

Let $\mathrm{B}=$ Base rate of the diagnostic category

Let $P(D)=$ Probability of being diagnosed as being maladjusted.

$=$ Probability of true positive + probability of false positive

$=$ (True positive base rate $\times$ Percent correctly identi-

fied $)+($ Negative Base Rate $\times$ Percent incorrectly identified $)$

$=(\mathrm{B} \times \mathrm{TP})+((1-\mathrm{B}) \times \mathrm{FP})$

$P(\mathrm{C} \mid \mathrm{D})=$ Probability of belonging to the category given diagnosis

$=P(\mathrm{D} \mid \mathrm{C}) P(\mathrm{C}) / P(\mathrm{D})$ [Bayes' Rule]

$=(\mathrm{TP} \times \mathrm{B}) /(\mathrm{B} \times \mathrm{TP})+((1-\mathrm{B}) \times \mathrm{FP})[$ Substitute $P(\mathrm{D})]$

$=(\mathrm{TP} \times 0.5) /(0.5 \times \mathrm{TP})+(0.5 \times \mathrm{FP})[$ Let the base rate $\mathrm{B}=0.5]$

$=\mathrm{TP} / \mathrm{TP}+\mathrm{FP}[$ Divide by 0.5$]$

Along with the extreme cases considered above (100\% or $0 \%$ base rates), this case of $50 \%$ base rates is another "degenerate" case of Bayes' Rule, in which the rule is not really needed. When the base rate of a disorder is $50 \%$, the conditional collapses to the simple (i.e., unconditional) probability that is given by the ratio of the probability of getting diagnosed correctly to the probability of getting diagnosed at all, whether correctly or not. One way of understanding what is happening in this case is to note that the true and false positive rates are sampling equally from the population. When this is so, we don't need to bother to "weight" their respective contributions to the conditional probability of belonging to the category given a diagnosis. 
A concrete example may make this interpretation more clear. Consider the conditional probability of having blue eyes, given that you are female. Since eye color is not a sex-linked character, the conditional is the same for both those who are in the group of interest (females) and those who are not (males). You may be able to intuit in this case that the conditional is therefore irrelevant: that is, the probability of being blue-eyed given that you are female is just the same as the probability of being blue-eyed.

This degenerate case of exactly equal base rates with and without the character of interest may occur only rarely, but the general principle illustrated by this case is of wider relevance for the reason note above: the further the positive and negative base rates are from being equal, the greater the difference between the conditional probability that depends on that base rate and the simple probability given by the ratio of the probability of getting diagnosed correctly to the probability of getting diagnosed at all (that is, the ratio of the true positives to the sum of the true and false positives).

Intuitively, this makes sense for the following reasons. Insofar as a disease is less common, it becomes more likely that a larger portion of the positives are false positives, as in the case considered above that bamboozled so many of the Harvard medical students. By the same token, insofar as a disease is more common, it becomes more likely that many of the negative diagnoses are false. At some point as base rates increase, they may come to exceed the ability of the test to identify them, rendering the test worse than guessing, as discussed above.

Bayes' Rule may be easily generalized to incorporate multiple pieces of evidence bearing on a single belief, hypothesis, or diagnosis, or to incorporate multiple pieces of evidence bearing on multiple beliefs, hypotheses, or diagnoses.

The simplest way to "extend" Bayes' Rule is to note that the posterior probability may depend on more than one piece of evidence. This is not an extension at all, since we noted at the beginning that what was given in a conditional may be a set of evidence rather than a single piece of evidence. However, it is worth emphasizing this point, since so many of the examples considered in this paper have treated the conditional as a single piece of evidence. Given a belief, hypothesis, or diagnosis $\mathrm{H}$, and a single relevant piece of evidence $\mathrm{E} 1$, we have seen how to compute some new probability $P(\mathrm{H} \mid \mathrm{E} 1)$. If we get a new piece of relevant evidence $E 2$, that is independent from E1, we could as easily calculate $P(\mathrm{H} \mid \mathrm{E} 2)$ for the same $\mathrm{H}$. However, that calculation would not take into account the fact that we already attached a certain level of probability to $\mathrm{H}$ because of the prior evidence A. To get that, we need to calculate $P(\mathrm{H} \mid \mathrm{E} 1 \& \mathrm{E} 2)$.

For example, imagining trying to guess a single card from a deck. If you know it is red, then you have $P$ (Guess $\mid$ Red $)=1 / 26$, because there are 26 red cards in a deck. If you know it is a face card, you have $P($ Guess $\mid$ Face $)=4 / 13$, because there are four face cards per suit of 13 cards. If you know it is both a face card and red, you need to calculate $P$ (Guess $\mid$ (Face $\&$ Red $)=8 / 52$ or $2 / 13$, because there are eight cards that are both red and a face card.

\section{REFERENCES}

Belmaker, R. H., Bersudky, Y., and Lichtenberg, P. (2010). Bayesian approach to bipolar guidelines. World J. Biol. Psychiatry 11, 76-77.
Butcher, J. N., Williams, C. L., Graham, J. R., Archer, R. P., Tellegen, A., BenPorath, Y.S., and Kaemmer, B. (1992). MMPI-A (Minnesota Multiphasic Personality Instrument-Adolescent):

A slightly more complex way of generalizing Bayes' Rule comes about when there is more than one competing hypothesis, diagnosis, or possibility to be considered. In that case, evidence brought to bear in favor of any single hypothesis needs to be considered in the context of the domain of all other competing hypotheses. In fact the simple forms of Bayes' Rule we have considered in this paper does exactly this. We have seen that $P(\mathrm{H} \mid \mathrm{E})=P(\mathrm{E} \mid \mathrm{H}) P(\mathrm{H}) / P(\mathrm{E})$, where $\mathrm{H}$ is some hypothesis, diagnosis, or possibility, and $\mathrm{E}$ is some evidence bearing on it. We have also seen in several examples that the denominator $P(E)$ - to be concrete, the probability of getting a positive diagnosis - can be expanded into sum of (the true positive rate $\times$ the positive base rate) and (the false positive rate $\times$ the negative base rate). The two elements in this sum are just two different hypotheses about where a positive diagnosis could have come from: it could either have come from a mistaken diagnosis or a true diagnosis. If there was also a possibility of a deliberately fraudulent diagnosis, we would have to add that in to our calculation of the probability of getting a positive diagnosis, as a third term in $P(\mathrm{E})$.

The generalization of Bayes' Rule to handle any number of competing hypotheses simply makes explicit that the denominator in Bayes' Rule is the domain of possible kinds of evidence that could explain $\mathrm{H}$ or said another way, the domain of possible ways the evidence under consideration could come about. The generalized expression is:

$P\left(\mathrm{H}_{\mathrm{n}} \mid \mathrm{E}\right)=P\left(\mathrm{E} \mid \mathrm{H}_{\mathrm{n}}\right) P\left(\mathrm{H}_{\mathrm{n}}\right) / \Sigma\left[P\left(\mathrm{E} \mid \mathrm{H}_{\mathrm{n}-1}\right) P\left(\mathrm{H}_{\mathrm{n}-1}\right)\right]$

$\mathrm{H}_{\mathrm{n}}$ is a current hypothesis, and $\mathrm{E}$ is, as ever, some new piece of evidence, such as a diagnostic sign. The denominator, as above in the specific cases we have considered, is simply the sum of all ways the diagnostic sign might occur, howsoever that may be.

\section{CONCLUSION}

Bayes' Rule has important implications for clinicians, allowing as it does for formal specification of the probability of a diagnosis being correct taking into account relevant prior probabilities. Although Bayes' Rule is simple, it is often ignored in practice, perhaps because the mathematics underlying the rule is often either dealt with in cursory manner in clinical training or else left under-specified. Although Meehl and Rosen's (1955) exposition of the importance of Bayes' Theorem is thorough and convincing, it left many proofs for the reader, with an apparent (probably erroneous) assumption that they were too simple to include. In this article I have followed the substance of Meehl and Rosen's exposition, but started from a simpler base and provided all the details of algebraic derivation that were left out of that article. My goal in doing so has been to make their exposition of Bayes' Rule more accessible, and thereby make it possible for more clinicians to benefit from their ground-breaking work demonstrating the importance of the rule in clinical settings.

\section{ACKNOWLEDGMENT}

Thanks to Gail Moroschan for feedback on an earlier draft of this article.

Manual for Administration, Scoring, and Interpretation. Minneapolis: University of Minnesota Press.

Fontaine, J. L., Archer, R. P., Elkins, D. E., and Johnsen, J. (2001). The effects of MMPI-A T-score elevation on classification accuracy for normal and clinical adolescent samples. J. Pers. Assess. 76, 264-281. 
Hacking, I. (2001). An Introduction to Probability, and Deductive Logic. Cambridge, England: Cambridge University Press.

Hart, S., Cox, D., and Hare, R. (1995). Manual for Psychopathy Checklist: Screening Version (PCL:SV). Toronto, Canada: Multi-Health Systems.

Kemp, S., Wilkinson, K., Caswell, H., Reynders, H., and Baker, G. (2008). The base rate of Wada test failure. Epilepsy Behav. 13, 630-633.

Meehl,P., and Rosen,A. (1955). Antecedent probability and the efficiency of psychometric signs, patterns, or cutting scores. Psychol. Bull. 52, 194-216. As reprinted in: Meehl, P.Psychodiagnosis: Selected Papers. New York, USA:W.W. Norton \& Sons; 1977.

Skeem, J. L., and Mulvey, E. P. (2001). Psychopathy and community violence among civil psychiatric patients: results from the MacArthur Violence RiskAssessment Study. J. Consult. Clin. Psychol. 69, 358-374.

Zanarini, M. C., Weingeroff, M. A., and Frankenburg, F. R. (2009). Defense mechanisms associated with borderline personality disorder.J. Pers. Disord. 23, 113-121.

Conflict of Interest Statement: The author declares that the research was conducted in the absence of any commercial or financial relationships that could be construed as a potential conflict of interest.

Received: 24 June 2010; paper pending published: 04 August 2010; accepted: 19 October 2010; published online: 16 November 2010
Citation: Westbury CF (2010) Bayes' rule for clinicians: an introduction. Front. Psychology 1:192. doi: 10.3389/ fpsyg.2010.00192

This article was submitted to Frontiers in Psychology for Clinical Settings, a specialty of Frontiers in Psychology.

Copyright (c) 2010 Westbury. This is an open-access article subject to an exclusive license agreement between the authors and the Frontiers Research Foundation, which permits unrestricted use, distribution, and reproduction in any medium, provided the original authors and source are credited. 